der Proteinkörpertherapie von Petersex in dem eingangs zitierten Werke durchgeführt worden. PeTersen führt aus, daß man verschiedene Arten von Serumenzymen unterscheiden müsse. Er teilt diese ein in zwei Leukoproteasen, die eine bei schwach alkalischer oder neutraler Reaktion, die andere bei schwach saurer Reaktion wirksam, beide befähigt, native Eiweißkörper bis zur Proteosen(Albumosen)Stufe zu spalten. Eine dritte Leukoprotease dagegen wirke wie ein Erepsin und spalte die Proteosen zu ungiftigen Aminosäuren. PETERSEN leitet diese Fermente vom Zerfall zerstörter weißer polymorphkerniger Blutkörper her und unterscheidet von ihnen endlich noch trypsinähnliche, die aus dem Magendarmkanal, den großen Abdominalorganen und pathologisch veränderten Herden stammten. Das Auftreten der die erste Spaltung bewirkenden Fermente könne sowohl primär giftige Eiweißkörper, welche die Eigenschaft hätten; den Körper zu sensibilisieren, entgiften, als andererseits zur Entstehung giftiger Abbauprodukte aus primär nicht giftigen Eiweißkörpern führen. Die Ereptase dagegen könne nur entgiftend wirken. Ich führe diese Ansichten Prerersens namentlich deswegen an, weil er sie auch in die Praxis überträgt. Er gibt z. B. Kurven der Ereptasenmenge und vergleicht sie mit der Temperaturkurve und der Kurve der Antikörper im Verlauf von Pneumonien mit dem Resultat, daß die Ereptasekurve und nicht die Antikörperkurve dem Verlaufe der Erkrankung entspräche. PETERSEN gibt in seinem Buche nur zusammenfassend die Resultate. Seine Methodik muB in seinen Einzelarbeiten nachgelesen werden. Die Wirkung dieser Fermente wird nach Petersen von Antifermenten reguliert und in einer Störung der Ferment-Antifermentbalance durch vermehrte Fermentbildung sieht Petersen das Wesen der Proteinkörpertherapie. Bemerkenswert ist, daß auch nach Petersen diese Antifermente keine Antikörper im immunologischen Sinne sind, sondern Lipoide. Ich hebe das hervor, weil Freund seine Serumgifte alkohollöslich fand. Freund und GotTlieb schreiben in ihrer letzten Arbeit: „Uber die chemische Natur der Zellzerfallprodukte, mit deren Zirkulieren wir die Reaktionsänderung in Zusammenhang bringen, kann man bisher nichts aussagen. Nur ihre Alkohollöslichkeit ist durch. Freund festgestellt. Man wird also an Lipoide z. B. an Phosphatide oder alkohollösliche Prođukte des Eiweißabbaues, vor allem an die proteinogenen Amine $\mathrm{zu}$ denken haben, dagegen wird durch den Befund der Alkohollöslichkeit die Beteiligung von höheren Spaltungsprodukten des Eiweißes, die sonst physiologisch sehr wirksam sind, unwahrscheinlich." Mehr läßt sich zur Zeit über die chemische Natur der wirksamen Stoffe in der Tat nicht sagen. Höchstens könnte es sich um verschiedene Körper mit verschiedenem chemischen Verhalten handeln.

Die Wirkungsweise des angenommenen, durch die Proteinkörper bzw. durch die unspezifische Therapie entstehenden Agens ist bekanntlich verschieden aufgefaßt worden. WEICHARDT sieht sie in einer Protoplasmaaktivierung und nennt das oder die verschiedenen primär wirkenden als Folge des therapeutischen Eingriffs im Körper entstehenden Agentien Protoplasmaaktivatoren. Er glaubt, daß es durch diese Protoplasmaaktivierung zu einer Leistungssteigerung aller Zellen käme, zu einer omnicellulären Wirkung. WEICHARDT hat ja auch eine erhöhte Funktion z. B. der Muskeltätigkeit oder der Milchsekretion als Folge der parenteralen Einverleibung von Eiweißkörpern gesehen. BrER hat an dem Ausdruck
Protoplasmaaktivierung Anstoß genommen und will ihn durch den Ausdruck Reiz im Sinne VIRchows ersetzen oder, um die Wichtigkeit der richtigen Dosierung zu betonen, durch den Ausdruck Schwellenreiz. BiERs Schüler ZIMmer spricht daher von Schwellenreiztherapie. voN GRöER hat den Ausdruck ,ergotrope Therapie" gewählt, um damit die Umstimmung der alten Autoren, die Veränderung der Reaktionsfähig-. keit zu bezeichnen. Er hält diese Wirkung nicht nur für omnicellulär, sondern auch für omnihumoral.

In der Tat ist ja eine Veränderung der Reaktionsfähigkeit, und zwar eine langdauernde Anderung besonders exakt durch die Arbeiten von GoTTLIEB ${ }^{26}$ ) und FREUND erwiesen worden. Sie sahen Änderungen in der Reaktionsfähigkeit des autonomen und sympathischen Systems, Veränderungen, die namentlich in den peripheren Endapparaten ihren Sitz haben, aber doch auch Anderungen der zentralen Erregbarkeit. Naturgemä $\beta$ können Veränderungen in der Erregbarkeit des vegetativen Systems omnicelluläre Wirkungen vortäuschen bei der großen Bedeutung dieses Systems für alle Lebensvorgänge. Das betonen GotTlIEB und FREUND auch mit Recht. Freund und GotTLIEB nehmen eine hormonartige Wirkung der als Folge der Eingriffe der unspezifischen Therapie entstehenden Stoffe an. Sie suchen also doch in Zellzerfallsstoffen und nicht in den Fermenten selbst das Wirksame. Man hat auch daran gedacht, da $B$ eine Zustandsänderung der Blutkolloide ganz allgemein gesprochen, wie sie durch das direkte oder indirekte Einströmen blutfremder Kolloide gegeben sei, einen von der Norm abweichenden pkysikalischen Zustand, eine erhöhte Labilität herbeiführen könne [SACHS ${ }^{27}$ )], und $\mathrm{daB}$ dieser die Zellfunktionen verändere. Das ist durchaus möglich, aber wir wissen darüber nichts Exaktes.

Theorien dieser Art werden uns nicht weiter helfen. Nur Beobachtung und Experiment werden die vielen und interessanten Fragstellungen der Lösung näher bringen, welche die Proteinkörpertherapie aufgibt. Ihre Einführung in die Praxis ist das bleibende Verdienst R. Schmints.

Literatur: 1) Schitrenhelm, Med. Klinik 1922, Nr. 30. 2) KazNelson, WeichardTs, Ergebn. d. Hyg. 4, 249. I920. 3) Stintzing, Münch. med. Wochenschr. I922, Nr. 7. - $\left.{ }^{4}\right)$ W. F. Perersen, Proteintherapie and non Specific Resistance, New York, The Macmillian Company ig22. - ${ }^{5}$ ) STARKenstein, Münch. med. Wochenschr. I9I9, Nr. 3, S. 8. - ${ }^{6}$ K KEHL,Arch f. exp. Pathol u. Pharmakol. 35, 222. - 7) Matrues, Dtsch. Arch. f. klin. Med. 54, 39. - 8) Matteres, Zentralbl. f. inn. Med. i 895, S. 285. 9) KREHI u. Matthes, Dtsch. Arch f. klin Med. 54, 50I. - ${ }^{10}$ ) KREHL u. Matthes, Arch. f. exp. Pathol. u. Pharmakol. 38, 437. 11) KREHL u. Matthes, Arch. f. exp. Pathol. u. Pharmakol. 40, 436. - 12) Martin, Arch. f. exp. Pathol. u. Pharmakol. 40, 453. 13) Krrcherim u. Tuczer, Arch. f. exp. Pathol. $\mathfrak{x}$. Pharmakol. 77, 387. - ${ }^{14}$ ) Gottstets, Dtsch. Arch. f. klin. Med. 94, 255. 15) Matrines, Dtsch. Arch. f. klin. Med. 95, 36I, 366. - ${ }^{16}$ ) LANGE, Dtsch. Arch. f. klin. Med. 94, 552. - ${ }^{17}$ ) MatTHes, Arch. f. exp.Pathol. u. Pharmakol. 49, I97 u. 5I, 442. - ${ }^{18}$ ) Matrhes, Zieglers Beiträge I893. - ${ }^{19}$ ) Matrues, Münch. med. Wochenschr igo2, Nr. T, S. 20. 20) Kirchierm u. Bötrner, Arch. f. exp. Pathol. u. Pharmakol. 78, 99. - 21) KLewitz, KongreB f. inn. Med.192 I. - 22) KirchHeim, Arch. f. exp. Pathol. u. Parmakol. 73, I39. - ${ }^{23}$ ) Werzes, Arch. f. exp. Pathol.' u. Pharmakol. 77, I49. - ${ }^{24}$ ) Kirchremim u. ReINicke, Arch. f. exp. Pathol. u. Pharmakol. 77, 4I 2. - ${ }^{25}$ ) KIRchHeim, Arch. f. exp. Pathol. u. Pharmakol. 74, 374. $-{ }^{26}$ ) FrEUnd, Arch. f. exp. Pathol. u. Pharmakol. 86, 266; 88, 39; 91, 272 u. Med. Klinik I920, S. $437 .-27$ ) GotTlieb u. Freund, Arch. f. exp. Pathol. u. Pharmakol. 93, 92. - ${ }^{28}$ ) SACHS, Therap. Halbmonatsheft I920, S. 373 u. 405.

\title{
ORIGINALIEN.
}

\section{ZUR FRAGE DER FUNKTIONSSTÖRUNG} DER NIERE.

\section{Von}

Prof. H. Zondex (klin. Teil), Dr. H. Petow und Dr. W. SIEBERT (physik.-chem. Teil).

Aus der I. med. Klinik der Universität Berlin. (Direktor: Prof. W. HIS.)

In der Nierenpathologie liegen zumeist Untersuchungen vor, die die Ausscheidungsfähigkeit der organischen harn- fähigen Substanzen betreffen. Soweit anorganische in Frage kamen, handelte es sich in der Hauptsache um die Bestimmung des Anions Chlor. Mit der Feststellung des Reststickstoffs und den sonstigen Methoden, welche die Anreicherung organischer und anorganischer Bestandteile im Blute anzeigen, konnte ermittelt werden, in welchen Fällen die Niere durchlässig ist und wann eine Nierensperre besteht. Unsere Kenntnis über das Wesen der Nierenfunktionsstörung und des mit der Nierensperre auftretenden Symptomenkomplexes der Urämie ist dadurch wenig gefördert worden. 
Wir haben seit längerem den Gedanken verfolgt, daß die Funktion der spezifischen Nierenelemente den gleichen physikalisch-chemischen Gesetzen unterliegen muß, wie die übrigen mit fein differenzierter Funktion begabten Zellen anderer Organe. Es ist durch zahlreiche Untersucher für das Herz (isoliert. Froschherz) gezeigt worden, daß die Funktion desselben gebunden ist an ein bestimmtes Mengenverhältnis der verschiedenen Kationen in der Nährflüssigkeit. (I. LOEB, ZWARDEMAKER, F. Kraus, S. G. ZondeK, I. C. Hamburger u. a.) So wissen wir, daß eine auch nur geringe Vermehrung von K.-Ionen das Froschherz in Diastole stillstehen läßt, während umgekehrt eine Anreicherung von Ca-Ionen systolischen Herz-Stillzustand zur Folge hat. Auch an anderen Organen tritt derselbe Antagonismus zwischen Calcium und Kalium zutage. Speziell für die Niere konnte I. C. Hamburger zeigen, daß eine Störung der Kationenbilanz das Ausscheidungsvermögen der überlebenden Niere für Glykose tiefgreifend beeinflußt. In Verfolg dieses Gedankenganges haben wir bereits vor mehreren Jahren begonnen, auf die Bedeutung der anorganischen Elemente und ihr Verhältnis zur Nierenfunktion Gewicht zu legen, ohne jedoch damals, nicht zuletzt mangels geeigneter Methodik, zu brauchbaren Resultaten zu gelangen (siehe H. ZoNDEK, Dtsch med. Wochenschr. I9I8, Nr. I5 u. Zeitschr. f. Klin. Med. Bd. 87, H. 5 u. 6). Eine geignete Methodik fand sich in den von KRAMER-Tistall angegebenen Mikroanalysen.

In Vorversuchen stellten wir für eine große Anzahl von nierengesunden Personen die normalen Werte für die im wesentlichen in Betracht kommenden Kationen im Blute fest und fanden in Ubereinstimmung mit anderen Autoren für Calcium sehr konstant als Höchstwert I0,7, als niedrigsten Wert Io,2 $\mathrm{mg}$ in $100 \mathrm{ccm}$ Serum; für Kalium im allgemeinen Werte zwischen $\mathrm{I} 6$ und $\mathrm{I} 8 \mathrm{mg}$ in $100 \mathrm{ccm}$ aus Plasma gewonnenem Serum. Das Plasmaserum wurde gewonnen, indem wir das Blut unter Paraffin. liqu. in paraffinierten Röhrchen auffingen, schnell abzentrifugierten und das Plasma, ehe die Gerinnung eingetreten war, von den Blutkörperchen trennten. Diesen Umweg wählten wir, weil wir häufig im Serum des spontan geronnenen Blutes Gesunder wechselnde Werte fanden, die meist höher waren als die des Plasmaserums. Es hat den Anschein, als ob während des Gerinnungsprozesses Kalium aus den roten Blutkörperchen austritt.

Unsere Fragestellung war zunächst folgende: Unterscheiden sich Nierenkranke mit gestörter renaler Funktion von solchen mit erhaltener Funktion? Unsere an einer großen Anzahl von Nierenkranken erhaltenen Befunde ließen erkennen, daß mit dem Auftreten funktioneller Störungen der Kaliwm- und Calciumspiegel im Blute sein normales Niveau verläßt. Es kommt zu einer Verminderung des Calciums und meist zu einer mäßigen Erhöhung des Kaliumgehaltes. Wenn man alle Erscheinungen, durch welche die renale Störung zutage tritt, berücksichtigt, wie Steigerung des Reststickstoffs, Hyposthenurie, Verlust des Verdünnungsvermögens, Kochsalzausscheidungsunfähigkeit usw., so kann man unsere Resultate dahin zusammenfassen: Ist die Funktionsstörung ausgesprochen, so zeigt sich eine Calciumverminderung im Blute bis zu $6 \mathrm{mg}$ (auch in Fällen ohne Hydrämie!) und eine Kaliumanreicherung bis zu etwa $24 \mathrm{mg}$. Ein für diese Untersuchungen besonders instruktives Krankheitsbild ist die Nephrosklerose in ihren beiden funktionell sehr verschiedenen Phasen. Die ohne Funktionsstörung verlaufende ,gutartige Hypertonie" weist in der Tat im Gegensatz zu der mit Beeinträchtigung der Nierenfunktion einhergehenden malignen Form der Nierensklerose normale Verhältnisse bezüglich des Kationenspiegels im Blute auf. Ist eine Störung der renalen Fähigkeiten nachweisbar, so is $t$ dies auch in einer eben beginnenden Störung des Ionengleichgewichtes im Blute $\mathrm{zu}$ erkennen. Auch in Fällen, wo mittels unserer groben Funktionsprüfungsmethoden noch keine Zeichen renaler Insuffizien $z$ nachgewiesen werden können, konnten wir im Blute bereits eine leichte Verschiebung der normalen Ionenverhältnisse feststellen, häufig in dem Sinne, daß der Calciumwert zunächst einmal das normale Niveau überschritt. Auch bei alsuten, mit Funktionsstörungen einhergehenden Glomerulonephritiden ließen sich. ähnliche Ionenverschiebungen im Blute wie bei der chronischen Erkrankung nachweisen. Bei einer 2ojährigen, an akuter diffuser Glomerulonephritis' leidenden Patientin, die eine nachweisliche Beeinträchtigung ihrer Nierenfunktion aufwies (Konzentrationsvermögen bis zum spez. Gewicht von IoI 7 , Wasserversuch $84 \mathrm{I} \mathrm{ccm}$ von rooo, Reststickstoff $49 \mathrm{mg}$ in $100 \mathrm{ccm}$ Blutdruck $180 / 120 \mathrm{~mm} \mathrm{Hg}$ ), fanden wir im Blute einen erniedrigten Calciumwert von $9,3 \mathrm{mg}$ und einen normalen Kaliumwert ( $7 \mathrm{mg}$ ). Als der Zustand sich nicht nur subjektiv, was Kopfschmerz, Neigung zu Erbrechen, schwere Abgeschlagenheit usw. betraf, sondern auch objektiv gebessert hatte, und der Blutdruck auf I25 mm $\mathrm{Hg}$ (maximal) herabgesunken war, war der Calciumspiegel des Blutes wieder bis auf IO,I $\mathrm{mg}$ in $100 \mathrm{ccm}$ angestiegen.

Hier erhebt sich nun die Frage, ob die veränderten Ionenverhältnisse im Blute etwa als Folgeerscheinung renaler Undurchlässigkeit aufzufassen sind. Für das Calcium dürfte das nicht in Frage kommen, da die Werte im Blute abnorm niedrig liegen und auch, wie der eine von uns (H. ZONDEK) bereits früher nachgewiesen hat, Calcium (ebenso wie Phosphorsäure) von Schrumpfnierenkranken ohne jede Verzögerung ausgeschieden wird. Aber auch das Kalium wird, wie wir durch mehrere Bilanzversuche zeigen konnten, von Schrumpfnierenkranken nicht retiniert.

Fragt man nach einer Erklärungsmöglichkeit für die von uns gefundenen Verhältnisse, so könnte man daran denken, daß infolge der Nierensperre die Löslichkeitsbedingungen des Blutes für die Kationen geändert seien. Die Löslichkeit des Calciums in einer Salzlösung von ähnlicher Zusammensetzung wie das Blut ist, wie RoNa und TAKAHASHI gezeigt haben, abhängig erstens von der $\left[\mathrm{H}^{+}\right]$und zweitens von der Menge von Bicarbonationen. Diese gesetzmäBige Beziehung wird ausgedrückt durch die Formel: $\mathrm{Ca}^{++}=$Konstante $\frac{\left[\mathrm{H}^{+}\right]}{\text {Bicarbonat }}$. Die $\left[\mathrm{H}^{+}\right]$wird im Organismus durch die besonderen physikalisch-chemischen Eigenschaften einiger Salze, besonders der Kohlensäure in ihren verschiedenen Zuständen, unter allen Umständen, also auch im Urämikerblut, konstant erhalten, und zwar nach der Formel: $\left[\mathrm{H}^{+}\right]=$Konstante $\frac{\mathrm{CO}^{2}}{\text { Bicarbonat }}$ Die Menge an Bicarbonat im Blut kann wechseln. Würden z. B. im Blut nicht flüchtige Säuren retiniert werden, so muB Bicarbonat zu Kohlensäure umgewandelt werden, die durch die Lungen ausgeschieden wird. Die Bicarbonatmenge würde somit sinken, d. h. die Alkalireserve vermindert sein, obgleich die $\left[\mathrm{H}^{+}\right]$dieselbe geblieben ist. In der Tat haben ELMENDORF, sowie STRAUB und MEIER mit modernen Methoden zeigen können, da $\beta$ mit gestörter Nierenfunktion eine Acidose in diesem Sinne einherzugehen pflegt. Eine Verminderung des Bicarbonats würde aber, nach der vorher angegebenen Formel, bei konstanter $\left[\mathrm{H}^{+}\right]$eine Vermehrung des $\mathrm{Ca}$ bedeuten. Wir fanden jedoch eine Verminderung der Gesamtcalciummenge, ein Resultat, daß mit der Annahme eine Acidose kaum vereinbar scheint. Untersuchungen zwecks Klärung dieser Frage sind im Gange und werden in kürzerem mitgeteilt werden könmen.

Der physikalisch-chemische Zustand des Calciums wird jedoch nicht nur durch die eben erörterten Beziehungen allein beeinflußt. Etwa $30 \%$ des Calciums sind im Serum an Proteine gebunden. Nun haben Freudenberg und GyörgY die interessante Tatsache entdeckt, daß diese Bindung des Calciums an Proteine von stickstoffhaltigen Substanzen, Aminen, Aminosäuren usw. verhindert werden kann. Das würde den Gedanken nahelegen, daß die von uns erhobenen Befunde in ursächlichem Zusammenhang stehen mit der Retention derartiger $N$-haltiger Substanzen, und daß die Eiweißkörper durch den Reststickstoff derartig verändert sind, daß sie weniger Calcium zu binden vermögen.

Für das Kalium sind die Verhältnisse weniger übersichtlich als für das Calcium. Die Möglichkeit eines Verständnisses aber scheint uns immerhin durch folgenden Gedankengang gegeben zu sein. Wie der eine von uns (PETOW) kürzlich in der Gemeinschaft mit RoNa (erscheint bịnnen kurzen 
in der Biochem. Zeitschr.) zeigen konnte, wird auch das Kalium unter gewissen Bedingungen an Eiweißkörper gebunden, und zwar unter solchen, únter denen das Calcium die Tendenz hat, sich von denselben zu lösen. Wenn eine Verallgemeinerung dieses Befundes erlaubt ist, so kommt man zu der Vorstellung, daß die Eiweißkörper immer dann Kalium in ihren Komplex aufnehmen, wenn ihre Ca-Bindung gelöst wird, d. h. eine Verminderung des an Proteine gebundenen Calciums würde automatisch mit einer Vermehrung des Kaliums verbunden sein. Auf die antagonistischen Beziehungen, die zwischen $\mathrm{Ca}$ und $\mathrm{K}$ sowohl kolloidchemisch als auch hinsichtlich des Einflusses auf die Organfunktionen bestehen, würde somit ein neues Licht fallen.

Unentschieden bleibt vorläufig die Frage: Ist die Verschiebung des Kationenspiegels im Serum Nierenkranker Folge einer Veränderung anorganischer Bestandteile oder eine Veränderung der Eiweißkörper durch gewisse Bestandteile des Reststickstoffes? Wie dem auch sei, jedenfalls muß eine derartige Störung der normalen Ionenbilanz die Funktionen der Gewebe und nicht zuletzt auch die der Nieren wesentlich beeinflussen. Eine Calciumverminderung und Kaliumanreicherung im Blute findet man übrigens auch bei der Tetanie (Mc-Callum, Tisdall u. Kramer, Hocoland). Es ist anzunehmen, daß es hier der Muskel ist, an dessen Zelle das normale Elektrolytengleichgewicht gestört ist, während es für das Wesen der renalen Insuffizienz und ihrer Folgeerscheinungen ausschlaggebend sein dürfte, daß hier neben den Geweben auch die Niere kolloidchemisch verändert ist.

\section{ÜBER CHIRURGISCHE KOMPLIKATIONEN BEI TRICHOCEPHALUS- UND OXYURISINFEKTION (INVAGINATION BEI TRICHOCEPHALUS- UND OXYURIS- TYPHLITIS, PERIPROKTITISCHE OXYURISABSCESSE).}

$$
\text { Von }
$$

Prof Dr. ANSCHÜTz.

Aus der Kieler cinirurgischen Universitätsklinik.

In der chirurgischen Literatur der letzten Jahre ist immer häufiger die Rede von den Nematoden (Ascaris, Oxyuris, Trichocephalus); mit Recht, denn sie sind - wenn auch im ganzen selten - weit häufiger Anlaß zu chirurgischen Erkrankungen, als man früher annahm. Beweglich, wie sie sind, entgingen und entgehen sie nur oft der Feststellung und die Krankheitsursache bleibt ungeklärt. Der Chirurg muß auf sie fahnden, dann wird er sie wohl öfters vor oder bei seinen Eingriffen finden, und diese Beiträge sollen dazu dienen, die Aufmerksamkeit mehr als bisher auf die Würmer als Krankheitsursache zu lenken.

Fall I. Trichocephalen als Ursache einer circumscripten Typhlitis und einer cöcocöcalen Invagination.

Ein bis dahin vollkommen gesunder $3^{8}$ jähriger Mann, der den Krieg mitgemacht, der im besonderen nie an Darmstörungen, Koliken, Jucken am After, Schwindel oder Blutarmut gelitten hat, erkrankte am 2I. VIII. I92 I ganz plötzlich mit Schmerzen in der Blinddarmgegend. Kein höheres Fieber, kein Erbrechen. Stuhlgang normal. Aufnahme 22. VIII. I921: Blühender Mensch in bestem Ernährungszustand. In der. Cöcalgegend etwa apfelgroße etwas bewegliche, pralle, schmerzhafte Geschwulst. Rectal o. B. Urin o. B. Temperatur 37,8. Puls 90.

Unter Annahme einer Appendicitis sofortige Operation: Klares Exsudat. Harte Geschwulst im Coecum, die sich nach Vorlagerung desselben als nicht vom Appendix - der vollkommen normal ist - ausgehend, sondern in der Darmwand sitzend erweist. Der Cöcalsack ist wie eingedellt, circumscript verhärtet, die Serosa an dieser Stelle injiziert und mit Fibrin belegt. Der Befund spricht für Tuberkulose oder Tumor. Deshalb ileocöcale Resektion, Vereinigung End zu Seit. 20. IX. I92 I Entlassung. Seither dauernd bestes Befinden.

Das sofort aufgeschnittene Präparat zeigte I. eine pilzartige, odematöse Tumorbildung im Cöcalsack, 2, etwa ein Dutzend Trichocephaien teils frei, teils lockerer, z. T. aber auch so fest in der Darmwand fixiert, daß sie mit der Pinzette nicht leicht entfernt werden konnten. In der ödematösen Schleimhaut keine UIcerationen oder
Blutungen. Auf dem Durchschnitt des gehärteten Präparates sieht man deutlich, daß eine Eindellung des Cöcalsackes in das Coecum besteht, es sich also um eine beginnende cöcocöcale Invagination handelt.

Mikroskopiseher Befund: Die Mucosa ist gedehnt, die Krypten stehen weit auseinander und sind verkürzt. Das interstitielle Gewebe zeigt eine mehr oder weniger dichte Infiltration mit eosinophiten Zellen, daneben auch Plasmazellen und spärliche Lymphocyten, nirgends aber polynucleäre neutrophile Leukocyten. Auch die Muscularis mucosae von eosinophilen Leukocyten durchsetzt. Submucosa ist stark ödematös, bis $z u 2 \mathrm{~cm}$ dick, verbreitert. Die Gewebslücken $z$. T. leer, $z$. T. mit Fibrin, z. T. mit dichten Ansammlungen eosinophiler Zellen angefüllt, namentlich in Mucosanähe stellenweise wie gepflastert mit ihnen. In dem aufgelockerten Gewebe sind auch reichlich Fibroplasten vorhanden. Auch in der aufgequollenen Ringmuskulatur dichte eosinophile Infiltration. In der Subserosa auffallende Gefäßerweiterung in deren Umgebung eosinophile Zellinfiltrate. Die Serosa zeigt vielfach Endothelverlust und Fibrinauflagerungen. Der Kopf des Parasiten steckt in der oberflächlichen Schicht der Mucosa. Es macht den Eindruck, als ob er eine Krypte durchbohrt und die benachbarten verdrängt oder arrodiert hätte. Ringsherum zahlreiche eosinophile Zellen, spärliche Lymphocyten. Riesenzellen sind nicht zu sehen. Sehr auffallend ist, daß die Epithelzellen der Krypten in der Umgebung des Parasiten eine starke eosinophile Affinität aufweisen, ohne daß Zelldegenerationen bestehen. Daneben stark erweiterte Capillaren und hier und da kleine Hämorrhagien im interstitiellen Gewebe.

Es handelt sich um den seltenen Fall einer wohl schon länger bestehenden ausgesprochenen eosinophilen Entzündung des Cöcalsackes infolge von Trichocephaleneinbohrung mit Invagination in das Coecum, die durch Spasmus oder wohl eher durch entzündliches Anschwellen der eingedellten Darmpartie entstanden ist. Wir kommen darauf beim nächsten Fall zurück.

Einen ganz analogen Fall von Invagination hat KAPPELER aus Askanazys Institut ${ }^{1}$ ) jüngst beschrieben, bei ihm waren aber offenbar weil der Darm erst später aufgeschnitten wurde - die Trichocephalen alle frei. Also in 2 Fillen der gleiche Befund einer eosinophilen Trichocephalustyphlitis mit cöcocöcaler Invagination.

Der alte Streit, ob die Trichocephalen sich in die Darmwand einbohren oder nicht, ist durch ASKANAZY ${ }^{2}$ ) definitiv für die Einbohrung entschieden. Die Würmer lösen sich nur sehr bald post mortem los, wandern weiter und werden deshalb nicht mehr gefunden. Neverliche sichere Beweise für die Einbohrung der Trichocephalen hat CHRISTOPHERSEN ${ }^{3}$ ) gebracht. An I 3 unter 37 sofort nach dem Tode formalinisierten Leichen sah er den Wurm fest in der Mucosa, aber nie weiter als bis zur Submucosa vordringend. Er fand wie KAPPELER und wir dieselbe hochgradige; weitgehende Infiltration der Darmwand mit eosinophilen Zellen, dieselbe hochgradige Eosinophilie des Epithels in der Umgebung des Wurmes, fand auch Lymphocyten und Plasmazellen und vermißte ebenso die polynucleären neutrophilen Leukocyten. Nur beschreibt er sehr große Riesenzellen, die weder KAPPELER noch wir feststellen konnten.

Unsere mikroskopischen Befunde sind gewiß auffallend, aber sie stellen doch nur einen höheren Grad der für das Eindringen von. Würmern charakteristischen lokalen Eosinophilie dar, d. h. einer durch die lokale Giftwirkung der Parasiten hervorgerufenen eosinophilen Entzündung. Die starke Einwirkung der Parasiten auf ihre Umgebung offenbart sich augenfällig in der eosinophilen Affinität des Dr üsenepithels. In späteren Stadien gesellen sich dann zu den. eosinophilen Zellen Lymphocyten und Plasmazellen und manchmal auch Riesenzellen hinzu, bei uns traten auch schon Fibroplasten auf. $\mathrm{Ob}$ die eosinophilen Zellen alle aus den Blutgefäßen ausgewandert oder z. T. aus dem Gewebe stammen, ist schwer zu entscheiden. Vorherrschend sind $\mathrm{m}$. A. die ersteren, bei der Stärke der Parasitenwirkung auf die Drüsenzellen kann man den letzteren Gedanken aber auch nicht ganz beiseite schieben.

Makroskopisch sichtbare Veränderungen hat CHristopherseN an den I 3 Trichocephalusdärmen nur einmal gefunden, und zwar einen kleinen $A b s c e B$ in einer Cöcalklappe, der Wurm saß aber ziemlich weit davon entfernt. GTRARD $^{4}$ ) sah eine Appendicitis bei Trichocephalen. WeINBERG ${ }^{5}$ ) das gleiche, er beobachtete sie außerdem bei Schimpansen und erzengte sie auch mehrfach experimentell bei ihnen mit und ohne Eiterung, manchmal fand sich dabei auch Bact. coli im Blut. Von Aimé GuTnarD ${ }^{6}$ ) u. a. ist der Wurm

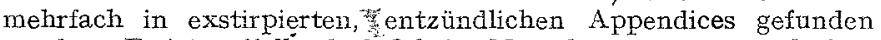
worden. Es ist auffallend, dab beim Menschen trotz massenhaften Einbohrens der Trichocephalen in die Darmwand polynucleäro Leukocytenanhäufungen, Eiterungen und Infektionen in der Regel fehlen. Trotz des sehr häufigen Vorkommens des Parasiten in 